\title{
Medição da densidade óssea através de ondas eletromagnéticas
}

\author{
Agnaldo Souza Cruz, Sandro Gonçalves da Silva e Bruno de Castro
}

\begin{abstract}
Resumo - Esse artigo apresenta uma proposta para medir a Densidade Mineral Óssea (DMO) como auxílio no diagnóstico da osteoporose. Um arranjo de antenas, afinado em uma frequência de uso geral e para aplicações médicas, é usado para medir a atenuação relativa do sinal em amostras de osso bovino, sílica e farinha de osso. Com a implementação de uma Máquina Vetor de Suporte (MVS), é possível estabelecer padrões para classificar a DMO como alta ou baixa.

Palavras-Chave - BMD, MVS, antena, osteoporose, atenuação.
\end{abstract}

Abstract - This article presents a proposal to measure the Bone Mineral Density (BMD) as a backup in the diagnosis of osteoporosis. An antenna arrangement, tuned to a frequency of general and medical use, is used to measure the relative attenuation of the signal on several samples of bovine bone, silica and bone powder. By implementing a Support Vector Machine (SVM) it is possible to establish patterns to classify the BMD as high or low.

Keywords-BMD, SVM, antenna, osteoporosis, attenuation.

\section{INTRODUÇÃO}

A cada trinta segundos uma pessoa sofre algum tipo de fratura em resultado da osteoporose (Figura 1). Uma em cada duas mulheres e um de cada quatro homens com mais de 50 anos terão fraturas relacionada à osteoporose. O Boletim da Organização Mundial de Saúde relata que uma cifra de $25 \%$ dos pacientes com 50 anos morrem por causa de complicações médicas causadas por fraturas ligadas à osteoporose [12]. Ela se tornou mais comum do que o colesterol alto, alergias e resfriado comum. Em geral, é silenciosa e não há sintomas de perda óssea até a ocorrência de fraturas.

A osteoporose é uma doença que se caracteriza por uma baixa massa óssea e deterioração na microarquitetura do tecido ósseo (Figura 1), levando a um aumento do risco de fraturas. Ocorre quando a massa óssea diminui mais rapidamente do que a capacidade do corpo de substituí-la, gerando a perda substancial da força do osso [12]. Ela afeta todos os ossos do corpo e não apresenta sinais ou sintomas até que uma fratura aconteça.

Há uma tendência de que o diagnóstico da osteoporose seja feito com base num certo nível de redução de massa óssea e não com base na presença de fraturas. A baixa densidade mineral óssea (DMO) é o fator de risco mais importante para o teste de Baixa Massa Óssea (BMD) [13].



Fig. 1. Efeitos da osteoporose.

Extensivos dados têm mostrado que a baixa massa óssea e o risco futuro de fraturas estão inversamente relacionados. A BMD é valiosa como previsor de fraturas assim como o colesterol alto ou pressão arterial elevada são previsores de infarto do miocárdio e acidente vascular cerebral respectivamente (OMS, 1994).

Dentre as técnicas disponíveis para determinação da DMO, tem-se a Dual Energy X-ray Absorptiometry (DEXA), a Tomografia Quantitativa Computadorizada (TQC) e a Ultrassonografia Quantitativa (QUS). As duas primeiras recorrem a feixes de raios $\mathrm{X}$ com diferentes níveis de energia e a terceira utiliza a velocidade e atenuação do som para medidas de DMO [12]. Na primeira descrição, tem-se a presença de radiação ionizantes que podem alterar ou destruir células do corpo humano. Já na segunda, embora não exista a presença de irradiação, há variabilidade entre os aparelhos utilizados, tendo-se assim variações nas medidas obtidas, sem a aplicação de critérios de diagnósticos clássicos empregados pela densitometria.

Este artigo tem por objetivo apresentar uma forma de medição da densidade óssea através da atenuação de ondas eletromagnéticas e posterior utilização da Máquina Vetor de Suporte para classificação da DMO. Para tanto, utilizou-se um arranjo de duas antenas de microfita com configuração retangular, operando a uma frequência de 2,49 GHz. Neste experimento, foram submetidas aos testes amostras de sílica, farinha de osso e osso bovino.

\section{ANTENAS}

Para o experimento, optou-se por um arranjo de duas antenas de microfita com configuração retangular (Figura 2), devido à facilidade de desenvolvimento, fabricação, características de radiação e construção simples de baixo custo [7].

Inicialmente, para construção das antenas, definiu-se a frequência de $2.44 \mathrm{GHz}$, alimentada por uma linha de microfita em um substrato de fibra de vidro com permissividade de 4,4 e altura (h) de 0,9 cm e dimensões descritas na Tabela I. 


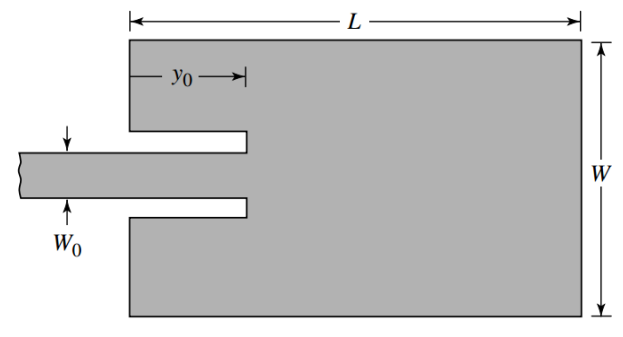

Fig. 2. Modelo da antena projetada (BALANIS,2009)

TABELA I. DIMENSÕES DA ANTENA PROJETADA

\begin{tabular}{l|l|l|l|l}
\hline $\mathbf{F}(\mathbf{G H z})$ & $\mathbf{W}(\mathbf{c m})$ & $\mathbf{L}(\mathbf{c m})$ & $\mathbf{Y}_{\mathbf{0}}(\mathbf{c m})$ & $\mathbf{W}_{\mathbf{0}}(\mathbf{c m})$ \\
\hline 2,44 & 3,74 & 2,92 & 0,969 & 0,172 \\
\hline
\end{tabular}

Os valores constantes da Tabela I foram obtidos aplicandose as seguintes fórmulas [7].

$$
\begin{gathered}
W=\frac{1}{2 f_{r} \sqrt{\mu_{0} \epsilon_{0}}} \sqrt{\frac{2}{\epsilon_{r}+1}}=\frac{v_{0}}{2 f_{r}} \sqrt{\frac{2}{\epsilon_{r}+1}} \\
\epsilon_{\text {reff }}=\frac{\frac{W / h>1}{2}+\frac{\epsilon_{r}-1}{2}\left[1+12 \frac{h}{W}\right]^{-1 / 2}}{\frac{\Delta L}{h}=0.412 \frac{\left(\epsilon_{\text {reff }}+0.3\right)\left(\frac{W}{h}+0.264\right)}{\left(\epsilon_{\text {reff }}-0.258\right)\left(\frac{W}{h}+0.8\right)}} \\
L=\frac{1}{2 f_{r} \sqrt{\epsilon_{\text {reff }}} \sqrt{\mu_{0} \epsilon_{0}}}-2 \Delta L
\end{gathered}
$$

As antenas foram projetadas e simuladas no software comercial Ansoft HFSS, com casamento de impedância ideal na frequência de 2,44 GHz (Figura 3).



Fig. 3. Perda de retorno - antena projetada no HFSS.

Após a confecção das antenas em laboratório (Figura 4) e medições realizadas no Analisador de rede Agilent - E5071C ENA (Figura 5) na prática, a frequência de ressonância ficou em $2,49 \mathrm{GHz}-2,05 \%$ de margem de erro da antena projetada.

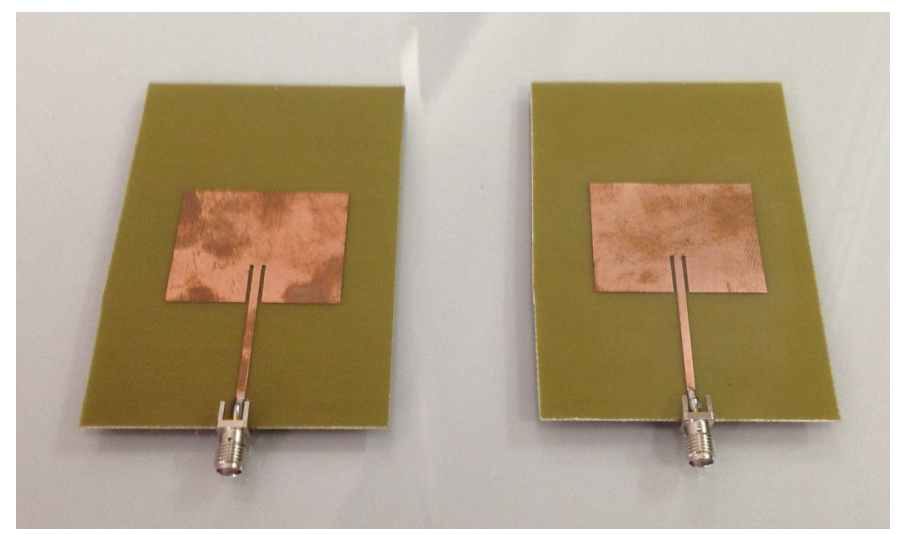

Fig. 4. Antenas confeccionadas em laboratório.

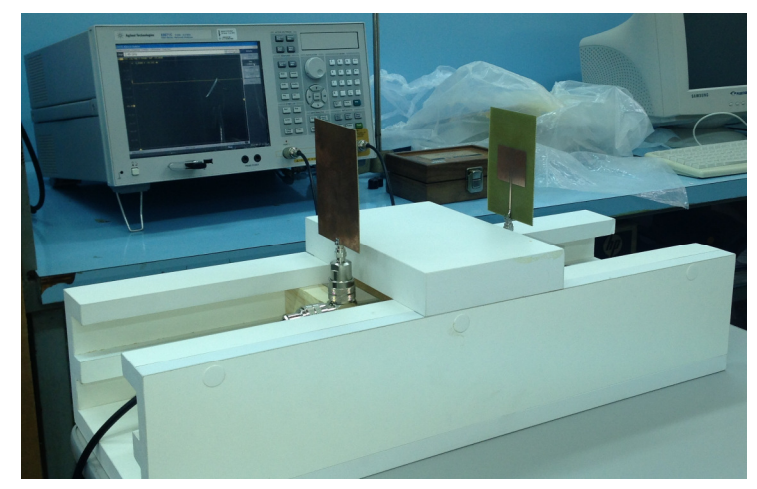

Fig. 5. Arranjo de antenas usado no experimento e Analisador de rede Agilent - E5071C ENA.

\section{SIMULAÇÕES - FARINHA DE OSSO E SÍLICA}

As simulações foram realizadas com quatro amostras de sílica e farinha de osso em quantidades alternadas adquiridas comercialmente. Como recipientes para as amostras, foram utilizados cilindros de acrílico com medidas de $6,0 \mathrm{~cm}$ de diâmetro e 6,6 cm de altura (Figura 6).

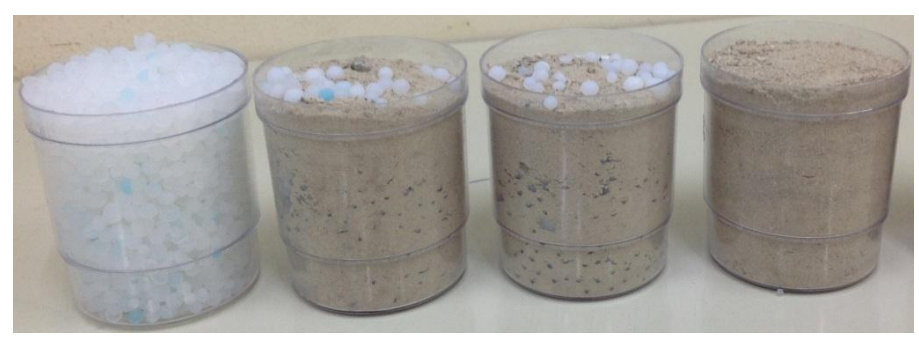

Fig. 6. Amostras de pó de osso e sílica.

A Tabela II exibe a variação do nível de atenuação em amostras com diferentes quantidades de farinha de osso e sílica, comprovando que as ondas eletromagnéticas emitidas na faixa de 2,49 $\mathrm{GHz}$ podem caracterizar as combinações de substâncias. 
TABELA II. AMOSTRAS DE PÓ DE OSSO E SÍLICA

\begin{tabular}{c|c|c|c}
\hline AMOSTRA & $\begin{array}{c}\text { OSSO } \\
(\mathrm{g})\end{array}$ & $\begin{array}{c}\text { SÍLICA } \\
(\mathrm{g})\end{array}$ & $\begin{array}{c}\text { ATENUAÇÃO } \\
(\mathrm{dB})\end{array}$ \\
\hline 01 & - & 79,16 & $-32,0114$ \\
\hline 02 & 182,67 & 0,00 & $-34,1453$ \\
\hline 03 & 104,42 & 37,65 & $-33,1704$ \\
\hline 04 & 141,54 & 17,30 & $-33,3856$ \\
\hline
\end{tabular}

\section{EXPERIMENTO - OSSO BOVINO}

Por reproduzir satisfatoriamente os aspectos de anomalia dos seres humanos, optou-se pelo uso de fêmures bovinos. Estes foram identificados com três grupos de A1, A2, A3; B1, B2, B3; C1,C2 e C3 para indivíduos distintos (Figura 7).

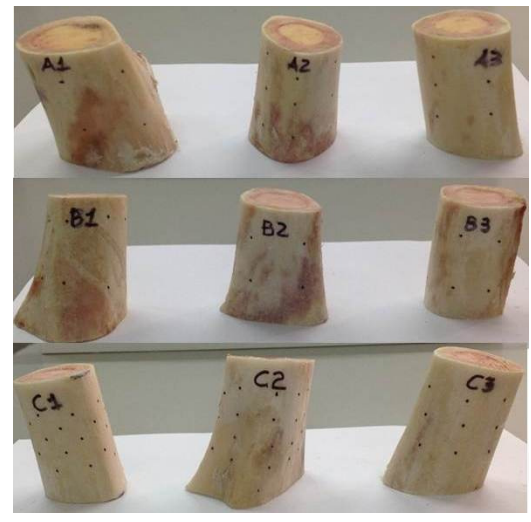

Fig. 7. Amostras de osso bovino utilizados.

Os ossos foram classificados por massa original e massa alterada (Tabela III). Após a pesagem, eles foram submetidos à radiação de microondas na frequência de $2,49 \mathrm{GHz}$ e foram verificados os seus respectivos níveis de atenuação.

TABELA III. MASSAS DE OSSOS ORIGINAIS E ALTERADAS

\begin{tabular}{c|c|c}
\hline OSSO & $\begin{array}{c}\text { ORIGINAL } \\
\mathbf{( g )}\end{array}$ & $\begin{array}{c}\text { ALTERADO } \\
\mathbf{( g )}\end{array}$ \\
\hline A1 & 204,2 & 198,6 \\
\hline A2 & 142,9 & 137,2 \\
\hline A3 & 145,3 & 141,0 \\
\hline B1 & 148,3 & 144,2 \\
\hline B2 & 139,3 & 133,1 \\
\hline B3 & 120,6 & 117,1 \\
\hline C1 & 123,0 & 120,2 \\
\hline C2 & 145,7 & 143,4 \\
\hline C3 & 132,6 & 123,4 \\
\hline
\end{tabular}

Realizada a descaracterização das amostras de osso, através de perfurações usando furadeira e brocas de diferentes diâmetros, procedeu-se com a pesagem e submissão à radiação na mesma frequência das amostras originais. Para cada amostra submetida à radiação, foram realizadas 200 leituras de sinais, totalizando 1800 sinais, sendo metade para massa óssea original e metade para massa óssea alterada.

Os resultados obtidos estão registrados na Tabela IV. Notase que as amostras originais apresentaram atenuação maior que as amostras alteradas com o processo de perfuração.
TABELA IV. ATENUAÇÃO MÉDIA DOS OSSOS

\begin{tabular}{c|c|c|c}
\hline OSSO & $\begin{array}{c}\text { ORIGINAL } \\
(\mathrm{dB})\end{array}$ & $\begin{array}{c}\text { ALTERADO } \\
(\mathrm{dB})\end{array}$ & $\begin{array}{c}\text { VARIAÇÃO } \\
(\mathrm{dB})\end{array}$ \\
\hline $\mathrm{A} 1$ & $-38,8783$ & $-38,6707$ & 0,2076 \\
\hline $\mathrm{A} 2$ & $-39,7907$ & $-39,5220$ & 0,2687 \\
\hline A3 & $-40,3946$ & $-40,3184$ & 0,0762 \\
\hline B1 & $-43,7795$ & $-42,8605$ & 0,9190 \\
\hline B2 & $-40,5919$ & $-39,9600$ & 0,6319 \\
\hline B3 & $-38,9521$ & $-38,8154$ & 0,1367 \\
\hline C1 & $-39,9055$ & $-39,8818$ & 0,0237 \\
\hline C2 & $-41,5019$ & $-40,8901$ & 0,6118 \\
\hline C3 & $-40,4362$ & $-38,9570$ & 1,4792 \\
\hline
\end{tabular}

\section{MÁQUINA VETOR DE SUPORTE}

\section{A. Definição}

A Máquina Vetor de Suporte (SVM - em inglês) é um algoritmo que possui a capacidade de construir um hiperplano (Figura 8) com superfície de decisão, definindo uma margem de separação entre dois exemplos, positiva e negativa. Ela aplica o principio da teoria da aprendizagem estatística, em que, considerando o caso de padrões separáveis, produz-se um valor de zero para o primeiro termo e minimiza-se o segundo [4]. Este algoritmo possui uma teoria mais complexa e rebuscada se comparada a outros algoritmos mais simples, como KNN.

As técnicas de aprendizagem de máquina utilizam um princípio chamado indução, no qual é possível atingir conclusões genéricas a partir das entradas fornecidas. Estas técnicas possuem dois tipos de aprendizagem: não supervisionado e supervisionado.

No primeiro, não existe a presença de um modelo previamente informado para aprendizagem da máquina. Ela aprende com a ajuda de uma medida de qualidade. Esta técnica é usada quando se tem por meta encontrar padrões em um conjunto de dados complexos. No segundo, que é a técnica de aprendizagem supervisionada, existe a entrada de um modelo, afim de que a máquina possa aprender. Têm-se as entradas e saídas conhecidas. A partir deste modelo, o algoritmo aprende com o objetivo de generalizar para posteriores entradas com as saídas esperadas.

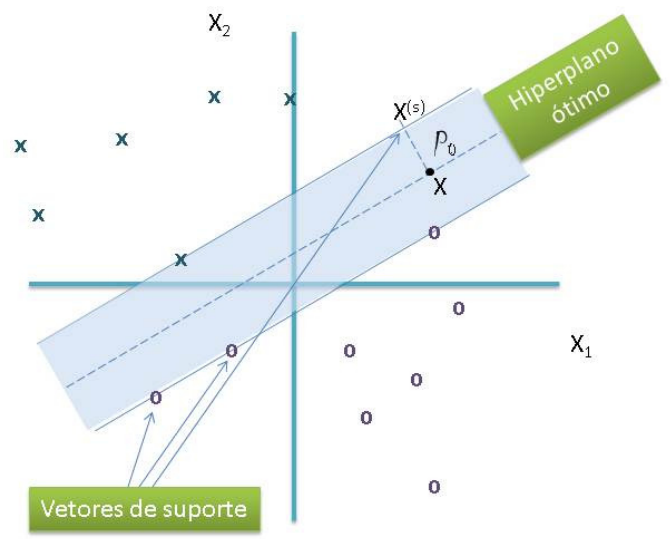

Fig. 8. Hiperplano ótimo para padrões separáveis. 
Em virtude disso, a SVM tem um bom desempenho de generalização em problemas de classificação de padrões, este sendo um atributo único das máquinas vetor de suporte. $\mathrm{O}$ objetivo é produzir um classificador que funcione de forma correta com exemplos que ainda não foram aplicados durante $o$ treinamento. Para tanto, é utilizado um recurso chamado de método de Kernel, que é capaz de produzir uma transformação para dar dimensionalidade aos dados, de forma a serem linearmente separáveis. Dentre os métodos disponíveis, foram utilizados neste experimento os métodos Linear, Polinomial e Radial Basis Function (RBF) [11].

Para o treinamento da SVM, foram separadas no total 900 leituras de sinais dos ossos A2, B3 e C1 com massa óssea original e medidas dos diâmetros superior, inferior, mediano e diâmetro da medula de cada amostra, usados para caracterização do indivíduo. A biblioteca utilizada para aprendizado de máquina foi a LibSVM [15] por ser otimizada para Máquina Vetor de Suporte, escrita em C na versão 3.17.

\section{RESUlTADOS}

Realizado o treinamento da SVM e posteriormente os testes com as amostras A2, B3, e C1 (TABELA V), originais e alteradas, foram obtidos os melhores resultados com classificação correta em $100 \%$ das amostras, com os métodos de Kernel Polinomial e Linear, atestando a eficiência destes.

TABELA V. OSSOS UTILIZADOS PARA TREINAR A SVM

\begin{tabular}{c|c|c|c|c}
\hline \multirow{2}{*}{$\begin{array}{c}\text { OSSO } \\
\text { ORIGINAL }\end{array}$} & $\begin{array}{c}\text { SUPERIOR } \\
(\mathrm{cm})\end{array}$ & $\begin{array}{c}\text { MEIO } \\
(\mathrm{cm})\end{array}$ & $\begin{array}{c}\text { INFERIOR } \\
(\mathrm{cm})\end{array}$ & $\begin{array}{c}\text { MEDULA } \\
(\mathrm{cm})\end{array}$ \\
\hline A2 & 4,0 & 4,4 & 4,5 & 2,4 \\
\hline B3 & 4,0 & 4,0 & 4,0 & 2,0 \\
\hline C1 & 3,6 & 3,6 & 3,6 & 2,0 \\
\hline
\end{tabular}

\section{CONCLUSÕES}

Conclui-se que, através de apenas uma onda não ionizante na faixa de 2,49GHz, é possível avaliar o nível de atenuação no tecido ósseo e, com a aplicação da Máquina Vetor de Suporte, alimentada com características obtidas no experimento, é possível classificar uma amostra como tendo densidade óssea baixa ou alta.
Pretende-se, para pesquisas futuras, estudar e aplicar processos de descaracterização das amostras pelo uso de ácidos, bem como ampliar o universo amostral, incluindo variáveis (a título de exemplo: tecido epitelial, conjuntivo e nervoso), com o objetivo de viabilizar o uso prático deste experimento.

\section{REFERÊNCIAS}

[1] GARDNER, Ernest; GRAY, Donald J.; RAHILLY, Ronan O'. Anatomia - Estudo Regional do Corpo Humano. Ed.4 Rio de Janeiro, RJ, 2008.

[2] MARTINI, Frederic H.; TIMMONS, Michael J.;TALLITSCH, Robert B. Anatomia Humana. Ed. 6 Porto Alegre, RS, 2009.

[3] MARTINI, Frederic H. Atlas do Corpo Humano. Ed. 6 Porto Alegre, RS, 2009.

[4] HAYKIN, Simon. Redes Neurais - Princípios e Prática. Ed. 2 - Porto Alegre, RS, 2008.

[5] SILVA, Ivan Nunes da; SPATTI, Danilo Hernane; FLAUZINO, Rogério Andrade. Redes Neurais Artificiais Para Engenharia e Ciências Aplicadas. São Paulo, SP, 2010.

[6] BALANIS, Constantine A. Teoria de Antenas - Análise e Síntese. Ed. 3, Vol 1, Rio de Janeiro, RJ, 2009.

[7] BALANIS, Constantine A. Teoria de Antenas - Análise e Síntese. Ed. 3, Vol 2, Rio de Janeiro, RJ, 2009.

[8] BARROS, Jannayna Domingues; OLIVEIRA, José Josemar Júnior; FARIAS, Robson Fernandes; SILVA, Sandro Gonçalves. Microwave Characterization Using Wavelets Na application related to bone tissue and bone meal with silica. MOMAG 2010.

[9] SADIKU, Matthew N. O. Elementos do Eletromagnetismo. Ed. 3, São Paulo, SP, 2004.

[10] JÚNIOR, João Bosco Amaral; JÚNIOR, José Lamartine Távora. Estudo Comparativo de Previsão Entre Redes Neurais Artificiais, Máquina de Suporte Vetorial e Modelos Lineares: uma aplicação à estrutura a termo das taxas de juros.

[11] CARVALHO, Lorena A. C.; Uma Introdução às Support Vector Machines. 2007.

[12] HENDERSON, Janet E.; GOLTZMAN, David; The Osteoporosis Primer. CAMBRIDGE UNIVERSIT PRESS, Cambridge, UK, 2000.

[13] National Osteoporosis Foundation. Clinician's Guide to Prevention and Treatment of Osteoporosis. Washington, DC: Nacional Osteoporosis Foundation; 2010.

[14] U.S. Departament of Health and Human Services. Bone Health and Osteoporosis: A Report of the Surgeon General. Rockville, MD: U.S. Departament of Health and Human Services, Office of the Surgeon General, 2004.

[15] CHANG, Chih-Chung; LIN, Chih-Jen; LIBSVM: a library for support vector machines. ACM Transactions on Intelligent Systems and Technology, 2:27:1-27:27, 2011. Software available at http://www.csie.ntu.edu.tw/ cjlin/libsvm

\footnotetext{
Agnaldo Souza Cruz, Sandro Gonçalves Silva e Bruno Henrique de Castro, Departamento de Engenharia Elétrica, Universidade Federal do Rio Grande do Norte, Natal-RN, Brasil, E-mails: agnaldo@ct.ufrn.br, sandro@ct.ufrn.br, bcastro@ct.ufrn.br.
} 